\title{
ICT Methodologies to Model and Simulate Parts of Human Body for Prosthesis Design
}

\author{
Giorgio Colombo ${ }^{1}$, Stefano Filippi ${ }^{2}$, Paolo Rissone ${ }^{3}$, and Caterina Rizzi ${ }^{4}$ \\ ${ }^{1}$ Politecnico di Milano, Dipartimento di Meccanica \\ Via G. La Masa, 34 - 20153 Milano, Italy \\ ${ }^{2}$ Università di Udine, DIEGM \\ Via delle Scienze, 208 - 33100 Udine, Italy \\ ${ }^{3}$ Università di Firenze, Dipartimento di Meccanica e Tecnologie Ind.li \\ Via S. Marta, 3 - 50139 Firenze, Italy \\ ${ }^{4}$ Università di Bergamo, Dipartimento di Ingegneria Industriale \\ Viale G. Marconi, 5 - 24044 Dalmine (BG)-Italy \\ giorgio.colombo@polimi.it, filippi@uniud.it, \\ paolo.rissone@unifi.it, caterina.rizzi@unibg.it
}

\begin{abstract}
The work presented in this paper refers to the implementation of a product development process based on the use of virtual model of the human body to design specific custom-fit product, such as a prosthesis socket (interface between the residual limb and the mechanical part of the prosthesis). It considers the integration of advanced ICT tools coming from the reverse engineering, the physics-based modelling and simulation, and the rapid prototyping fields. The paper describes problems related to the implementation of each step within a real socket development process.
\end{abstract}

Keywords: Product customization, Prosthesis design, Physics-based simulation, Human body modeling.

\section{Introduction}

Custom-fit products, especially those with an interface with the human body, cannot be completely designed using methods and tools developed for other industrial fields (i.e., mechanical field) for mass production or for modular products, but it is necessary to realize "ad hoc" methodologies.

The work presented in this paper refers to this context with the aim of verifying the implementation of a design paradigm for a specific custom-fit product, a prosthesis component, through the integration of advanced ICT tools, allowing the evolution from a hand-made production to a computer assisted realisation of highly customised products. We consider the case of the socket (interface between the residual limb and the mechanical part of the prosthesis) both for trans-femoral or trans-tibial amputees since it requires a high level of customisation. The socket plays the fundamental role in the comfort and functionality of the prosthesis. It has to be manufactured in the correct way both from anatomical and biomechanical point of view because each movement between stump and socket can hugely reduce the prosthesis control causing unsafe and pain during walking. 
The research has been developed in the framework of an Italian PRIN (Research Project of National Interest) Project named DESPRO (Integration of Innovative Methodologies to DESign and Develop Custom-fit Products: Application and validation for a Socket of a Lower Limb PROsthesis) funded by Italian Research Ministry. The consortium comprises four universities, University of Bergamo, Florence, Udine, and Polytechnic of Milan collaborating with an Italian prosthesis manufacturer named Centro Protesi INAIL, Budrio (BO).

\section{Traditional Process and Knowledge Acquisition}

Before proceeding with the implementation of the new design paradigm, we studied the traditional process followed by orthopedic technicians to design non-standard components of lower limb prosthesis. The product and process knowledge has been acquired analyzing scientific literature and through interviews with domain experts (medical, paramedical and technical staff) and with the patients that will wear the prosthesis. This activity has been particularly important since it permitted to define key characteristics of the socket and consequently possible improvements both for the product and for the design process.

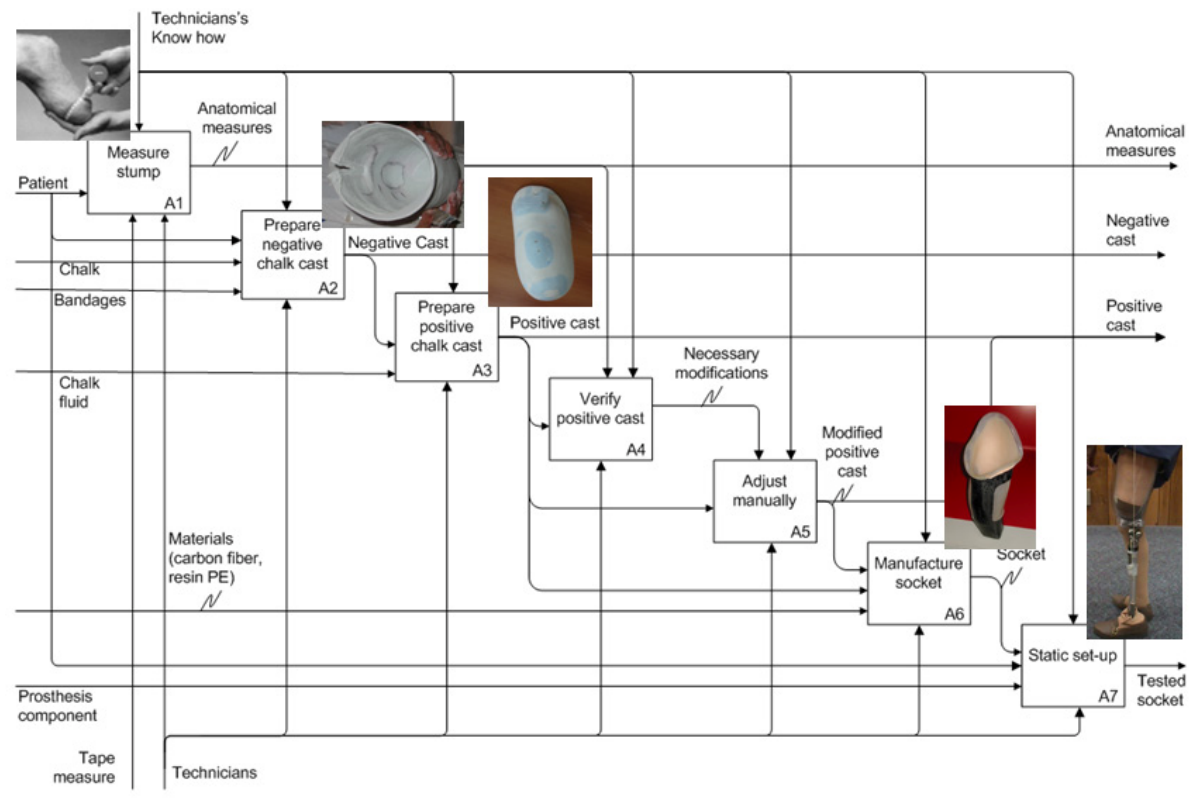

Fig. 1. Design and manufacturing process of a socket

First, the design and manufacturing of high-quality sockets must fulfill the following principles: accurate measurement of the stump geometry, perfect close fitting of the prosthesis to the stump, good response to forces and mechanical stress, 
safety, and each single area of the socket must have a tight connection to the stump anatomy without affecting blood circulation.

Both trans-tibial and trans-femoral sockets are manufactured starting from a positive chalk cast. The cast can be manufactured following a fully hand-made procedure or partially based on digital tools, such as CAD/CAM systems. In the last case, the procedure for trans-tibial and trans-femoral is slightly different but the output is always the 3D model of positive cast for the CAM system and, thus, to produce the physical prototype of the positive cast. However, in Italy, most of laboratories producing prostheses are SMEs and the design and manufacture of a socket is almost a hand made activity carried out by skilled technicians. Figure 1 portrays the main phases of the As-Is production process represented with and IDEF0 diagram [1].

In literature, we can find various researches on ICT tools to support some single steps of the process [2-11]; however, there is not a validation of a complete process based on digital data and tools. This highlights the need of new design methodologies that take into account materials used for the socket, the stump structure (i.e. bones, muscles and skin) and manage their mutual interactions.

\section{The New Development Process}

We propose a new design paradigm based on the use of virtual models of human body to design specific custom-fit product, such as the prosthesis socket. In such a context, different issues related to the human body should be considered, e.g., the acquisition of stump morphology, generation of a complete virtual model that includes both the external shape (skin) and the geometry of internal parts (muscles and bones) and mechanical characterisation of the stump to be able to simulate correctly the socketstump interaction.

Figure 2 shows the new design paradigm completely based on computer-aided tools and on the modelling and simulation of the two interfacing parts (human body and socket). It has been thought to implement best practices used by orthopaedic technicians and finalised to ensure high-level products independently of the competencies of the domain expert that manufactures the socket. It consists of five main steps, where following tools are integrated (Figure 3):

- reverse engineering tools for the automatic (or semi-automatic) acquisition of patient's morphology and bony-muscular structure (in our case the residual limb);

- a physics-based modeler allowing the designer to represent both the human body's parts and the socket as composed by different materials;

- $\quad$ an environment for physics-based simulation to reproduce the real behaviour of socket-stump system and to verify the product functionalities;

- Rapid prototyping tools for the realization of physical prototypes to test and validate the virtual product and to identify adjustments.

The first activity is devoted to the acquisition of the stump geometry for the reconstruction of its digital model and the corresponding socket model. Since ten years ago, several non-contact reverse engineering techniques and medical imaging 


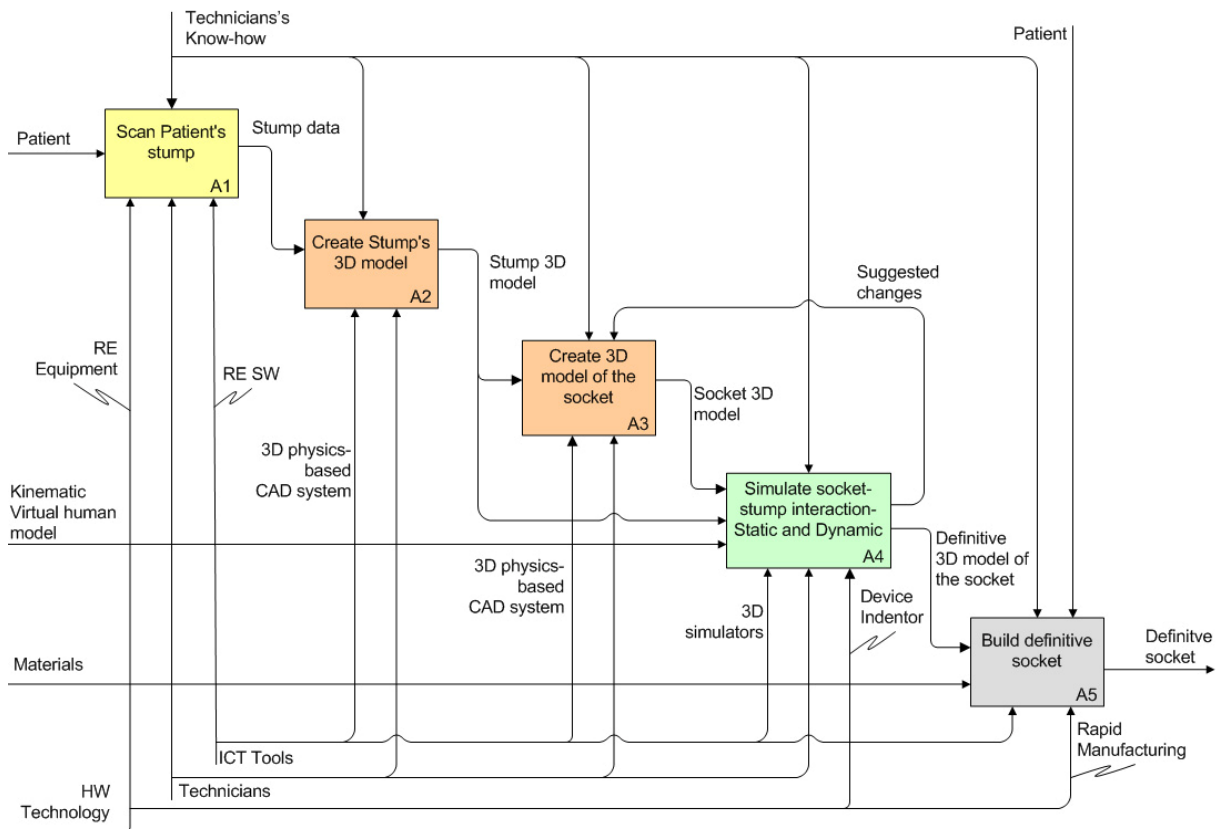

Fig. 2. The new product development process

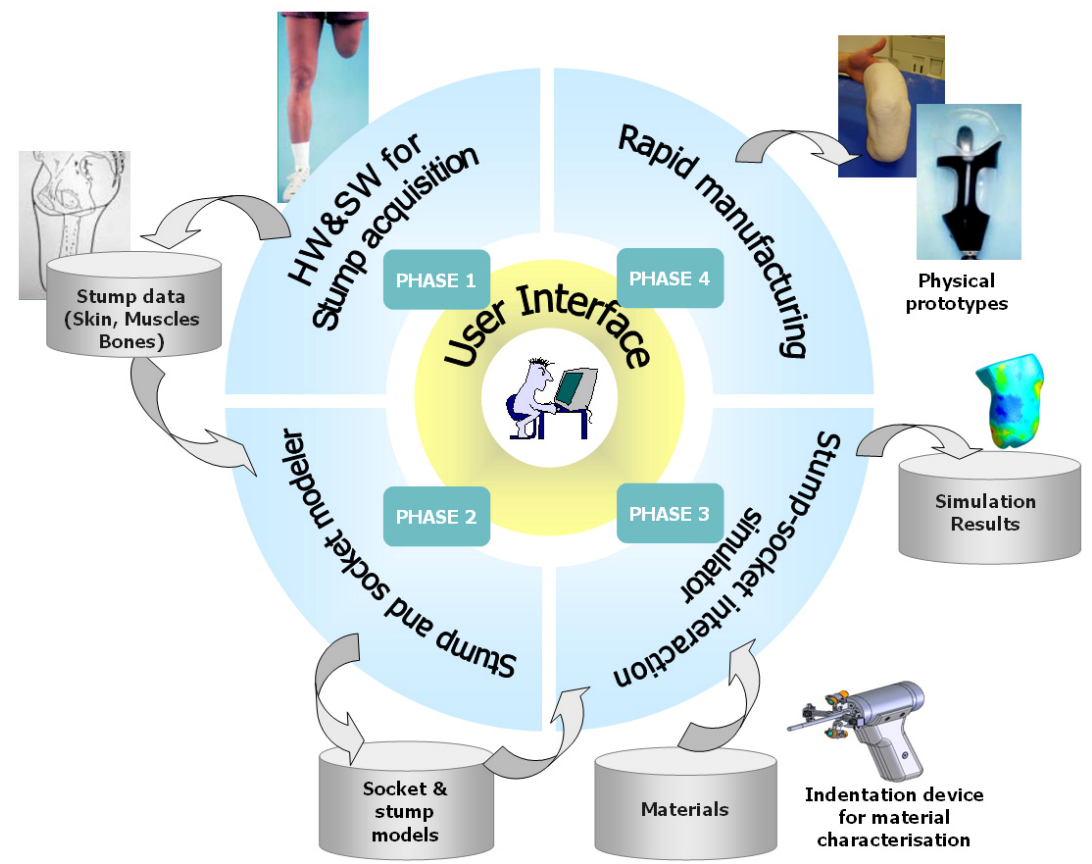

Fig. 3. The design process phases and related tools 
have been tested. First techniques for digitalizing the 3D geometry of the residual limb were based on the acquisition of bi-dimensional silhouettes [2], but more recently, MRI (Magnetic Resonance) and CT (tomography) have been considered in order to build a model as a set of cross-sectional images, by distinguishing bones, soft tissue and skin of the residual limb [3], [4], [5]. We adopted both reverse engineering based on laser technology and medical imaging techniques to obtain a model that includes both stump external shape and its inner parts (muscles and bones).

Concerning stump and socket modelling, CAD/CAM systems available on the market for prosthesis design (e.g., TracerCAD - www.tracerCAD.com, Ossur CAD www.ossur.com) permit to model the positive cast model and are not able to model objects made by different materials (e.g., for the stump: skin, tissue, bones...).

Therefore, the choice of the product model representation is one of the most important aspects of the whole project. In fact, the representation used to codify both the geometric information and the physical-mechanical features of the product can heavily influence the following simulation and manufacturing phases. Several modelling techniques and representation schemes have been considered; the choice has been the physics-based modelling (both the Particle-based modelling and Finite Element Method) [6], [8], [13] for what concerns the simulation activities and the parametric solid modelling for the rapid prototyping activities.

The aim of the simulation phase is to optimize the shape of the prosthesis socket according to fitting and wearability criteria. The simulation of the stump-socket biomechanical interaction is a very difficult task since the tissues of the residual limb have a strong non-linear mechanical behaviour and exhibit very large deformations under the loads that the stump experiences during the gait cycle. Particle-based [6] and the Finite Element [12] approaches have been investigated. While particle-based approach has been widely used only for real-time simulation purposes, the Finite Element Method has been adopted to simulate the interaction between the prosthesis socket and the stump according to the tasks of this work. However, the use of this method is still strongly limited by the material characterisation adopted to simulate the behaviour of the soft tissues. The optimization task requires material models that take into account the non-linearity of the human soft tissues and their distribution around the bones of the stump [9], [14], and [15]. According to the purposes of the road - map developed in this work, the authors are developing a device to measure the mechanical characteristics of the stump to characterise the material model for the simulation task.

Finally, rapid Prototyping (RP) can play an important role in this process reducing the time to deliver the definitive socket to the patient. Countless are the applications of RP in the medical field, specifically regarding prosthesis development, and last ones have been mainly considered for the production of the stump positive plaster cast and for socket manufacturing [10], [11]. In the context of this project, the effort is directed towards two main strategic directions: the application of the RP technologies and the re-engineering/optimization of the product oriented to the RP technology, aiming at the generation of the physical prototypes of the anatomical parts and the artefacts. 


\section{Design Process Experimentation}

Main efforts have been spent to demonstrate the practical implementation of the proposed approach and highlight the aspects to be improved. In the following, we will describe preliminary results of experimentation carried out until now with reference to each step of the new design paradigm. Both trans-tibial and trans-fermoral amputees have been considered; however, the complete process has been mainly validated for trans-tibial prosthesis.

\subsection{Geometry Acquisition}

A non-contact laser scanner (Minolta Vivid VI-9i ${ }^{\mathrm{TM}}$ ) has been adopted to acquire the external shape of the stump. We also acquired the geometry of the related positive plaster cast to compare the stump external shape with the socket internal surface and determine critical zones. Computer Tomography (CT) and Magnetic Resonance Imaging (MRI) have been adopted for the internal structure, respectively for bones and soft tissues and muscles. During acquisition, patient's posture has been determined using a support device that permitted to maintain lower limb position adopted for manual measurements. In addition, markers have been used to identify anthropometric standard points. For further details, see [16].

Figure 4 shows the geometry acquired for a trans-tibial amputee: (A) point clouds, (B) STL from CT, and (C) a MRI image with markers.
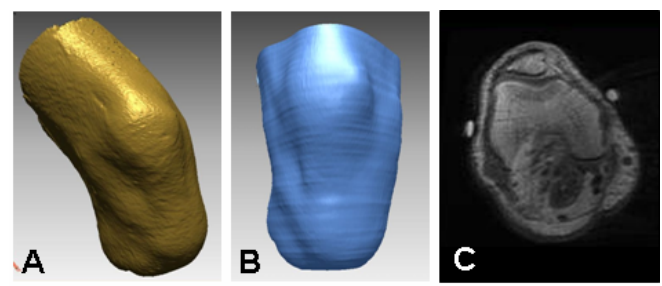

Fig. 4. Acquisition of stump external and internal shape

\subsection{Stump and Socket Modelling}

The stump geometric model has been generated integrating the data acquired through the three mentioned technologies [16]. Two types of geometric model have been reconstructed: one tasseled (STL) and another based on NURB surfaces.

The geometric model of the skin, derived from laser points clouds, ensures a high quality in morphological details necessary for the simulation of stump-socket interactions. For the internal parts both CT and MRI data have been used. CT and MRI could be considered quite similar from the shape reconstruction point of view. They are based on different technologies (X-ray and magnetic resonance of particles respectively) but both have as output a data volume consisting in a set of images, strictly ordered, and representing anatomical sections. Starting from this data, the 3D reconstruction of the anatomical structures is quite straightforward. 
The three different models have been assembled aligning them into the same reference global system taking into account markers used during acquisition phase (Figure 5a).

Regarding the socket model, we initially reconstructed it from the points of clouds acquired for the positive plaster cast (Figure 5b). Twice are the objectives: first compare the stump and socket model in order to identify main differences and, then, provide reference data to evaluate simulation results.

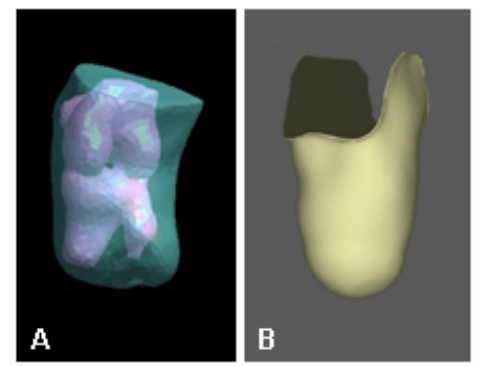

Fig. 5. Geometric models: a) stump model, b) socket model

\subsection{Stump-Socket Simulation}

The authors consider this task as a fundamental step to find the optimal morphology of the socket. We analysed two different aspects: the wearability (donning simulation) and patient's motion (gait simulation). By the donning simulation, the designer can verify if the shape of the socket allows the wearibility. In this way the presence of dangerous undercuts, which can produce stresses and large deformations of the soft tissue, can be easily identified. The gait simulation allows the analysis of the biomechanical behaviour of the socket-limb interface during the patient's walking. We first focused the attention on donning simulation adopting FE approach.

2D and 3D models, implicit and explicit codes and different types of material models are under investigation. A hybrid method based on indentation tests and Finite Element analysis has been developed to characterize the material models of the stump soft tissue. This allows defining ad hoc material models according to the anatomy of the residual limb of each patient so that more accurate results in the pressure distribution can be expected. Two different types of simulation are under investigation. The first, based on 2D elements, analyses meaningful sections of the stump and the socket. The second uses 3D elements and studies the complete interactions between the two mentioned parts. When the 2D simulations furnish good results, the second more complex simulation based on $3 \mathrm{D}$ models permits to verify the fitting and the wearibility of the whole $3 \mathrm{D}$ socket. If these verifications are satisfied, final model of the socket is reached. Otherwise, other modifications to the $3 \mathrm{D}$ geometrical model of the socket are planned and a new cycle of $2 \mathrm{D}$ and $3 \mathrm{D}$ simulations is performed.

In the approach based only on 3D model, the fitting and the wearibility simulations are performed without any preliminary 2D simulation. The task aims at verifying if the designer and the orthopaedic technician are able to optimize the socket shape 
dealing directly with 3D more complex geometrical models. In order to speed up the optimization of the socket shape the integration of optimization tools in the simulation task is, also, under investigation.

Figure 6 shows an example of a preliminary simulation of wearibility realized by using a 3D mesh and the explicit solver LS-DYNA; in this case, the bucket sortsearching algorithm is used as contact model between the socket and the limb.

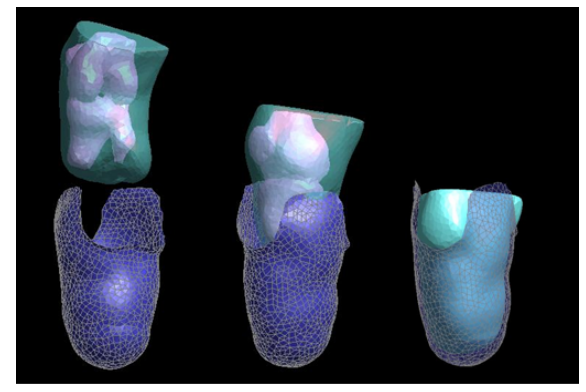

Fig. 6. Wearibility simulation

Figure 7 shows the pressure distribution obtained by a preliminary 3D simulation of the fitting between the socket and the stump under the body weight loads.

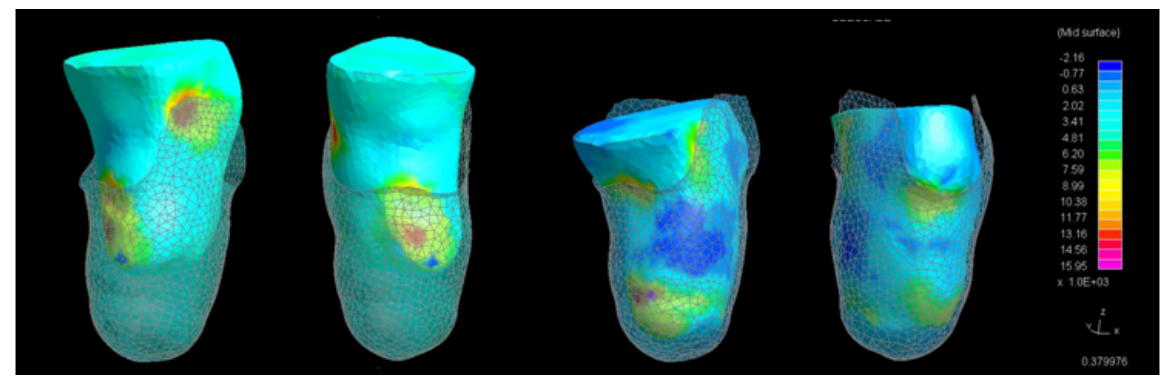

Fig. 7. Pressure distribution

For the simulations shown in Figures 6 and 7, only linear models have been used; for the bones, one with Young's modulus equal to $10 \mathrm{GPa}$, for the socket, a second linear elastic material with Young's modulus equal to $1.5 \mathrm{GPa}$, and, for the soft tissues, a third linear model with Young's modulus equal to $0.2 \mathrm{GPa}$ according to [17].

\subsection{Rapid Prototyping}

For what concerns the rapid prototyping, the activities now are focused on the definition of the product model, using the data coming day by day from the simulation phase. In a short time, the model of the first socket designed inside the framework described in this paper will be translated into the STL format and the best rapid 
prototyping technology will be selected and used to generate the physical representation. We think to generate a SLA model of the socket and to replicate it using vacuum casting. As soon as these models are available, the testing phase will take place to validate the design and engineering choices and to compare the results with those ones coming from the simulations. Based on a recursive algorithm, the simulation packages will be fine-tuned, to get the result of avoid the rapid prototyping phase and generate a correct result already at the simulation stage.

\section{Conclusions}

This paper describes a new design process based on the use of virtual humans and specifically conceived for a prosthesis component, the socket. Different ICT technologies, from reverse engineering to physics-based simulation and rapid prototyping, must be integrated to evolve from a hand-made approach to a new one computer assisted. The proposed design paradigm has been experimented both for trans-tibial and trans-femoral amputees and main problems related to the implementation of each step have been generally discussed. Problems related to stump measurement and modeling have been completely identified, and results from acquisition and modelling of the stump and of positive plaster cast provided useful guidelines for socket modelling and physics-based simulation. However, even if results obtained encourage us to proceed, some issues are still open and require further efforts, especially for the socket of trans-femoral amputee. Future research activities will be concentrated in particular on 3D socket modelling, the evaluation of more proper nonlinear models of material for highly deformable tissues, (e.g., hyperelastic and viscoelastic models). Finally, the feasibility and usefulness of simulations using "virtual human" will be considered and to estimate the effects of prostheses modifications

Aknowledgements. The authors would like to thank Massimiliano Bertetti, Daniele Bonacini and Grazia Magrassi from Politecnico di Milano for their invaluable research work on stump modeling, Barbara Motyl from Università di Udine and, Federico Rotini and Francesco Saverio Frillici from Università di Firenze.

\section{References}

[1] http://www.idef.com

[2] Schreiner, R.E., Sanders, J.E.: A Silhouetting Shape Sensor for the Residual Limb of a Below-Knee Amputee. IEEE Transactions On Rehabilitation Engineering 3(3) (1995)

[3] Santosh, G.Z., Sanders, J.E., Turkiyyah, G.M.: Automated Hexahedral Mesh Generation from Biomedical Image Data: Applications in Limb Prosthetics. IEEE Transactions On Rehabilitation Engineering 4(2) (1996)

[4] Ming, Z., Mak, A.F.T., Chung, A.I.K., Chung, K.H.: MRI investigation of musculoskeletal action of transfemoral residual limb inside a prosthetic socket. In: Proceeding 20th Annual International Conference of the IEEE Engineering in Medicine and Biology Society, Part 5/6, October 29-November 1, 1998, Hong Kong, China, pp. 2741-2743 (1998) 
[5] Shuxian, Z., Wanhua, Z., Bingheng, L.: 3D reconstruction of the structure of a residual limb for customising the design of a prosthetic socket. Medical Engineering \& Physics (27), 67-74 (2005)

[6] Nedel, N.P., Thalmann, D.: Real Time Muscle Deformations using Mass-Spring Systems. In: Computer Graphics International (1998)

[7] Lee, W.C.C., Zhang, M., Jia, X.: Load transfer mechanics between trans-tibial prosthetic socket and residual limb dynamic effects. Journal of Biomechanics 37, 1371-1377 (2004)

[8] Lee, W.C.C., Zhang, M., Jia, X., Cheung, J.T.M.: Finite element modelling of the contact interface between trans-tibial residual limb and prosthetic socket. Medical Engineering \& Physics (26), 655-662 (2004)

[9] Tönuk, E., Silver-Thorn, M.B.: Nonlinear viscoelastic material estimation of lower extremity residual limb tissues. Journal of Biomechanical Engineering 126(2), 289-300 (2004)

[10] Cheng, T.K., Sin, P.L.V., Fye, T.K., Lin, L.S.: Automation of prosthetic socket design and fabrication using computer-aided-design/computer-aided-engineering and rapid prototyping techniques. In: Proceeding First National Symposium on Prosthetics and Orthotics. Singapore (1998)

[11] Freeman, D., Wontorcik, L.: Shererolithiography and prosthetic test socket manufacture: a cost/benefit analysis. Journal of Prothestics and Orthotics 10(1) (1998)

[12] Mollemans, W., Schutyser, F., Cleynenbreugel, J.V., Suetens, P.: Tetrahedral mass spring model for fast soft tissue deformation. LNCS, vol. 2673(1), pp. 145-154. Springer, Heidelberg (2003)

[13] Faustini, M.C., Neptune, R.R., Crawford, R.H.: The quasi-static response of compliant prosthetic sockets for transtibial amputees using finite elements methods. Medical Engineering \& Physics N. 28, 114-121 (2006)

[14] Tönuk, E., Silver-Thorn, M.B.: Nonlinear elastic material property estimation of lower extremity residual limb tissues. IEEE Transaction On Neural Systems And Rehabilitation Engineering 11(1), 43-53 (2003)

[15] Zheng, Y.P., et al.: State - of - the - art methods for geometric and biomechanical assessments of residual limbs: a review. Journal of Rehabilitation Research and Development. 38(5) (2001)

[16] Colombo, G., Bertetti, M., Bonacini, D., Magrassi, G.: Reverse Engineering and rapid prototyping techniques to innovate prosthesis socket design. In: Proceeding SPIE-IS\&T Electronic Imaging, San Diego, CA, vol. 6056, 60560P (2006)

[17] Lee, W.C.C., Zhang, M., Jia, X., Cheung, J.T.M.: Finite element modelling of the contact interface between trans-tibial residual $\mathrm{limb}$ and prosthetic socket. In: Medical Engineering \& Physics N. 26, 655-662 (2004) 